Research Article

\title{
Gestational Weight Gain Intervention Impacts Determinants of Healthy Eating and Exercise in Overweight/Obese Pregnant Women
}

\author{
Abigail M. Pauley $\mathbb{D}^{1},{ }^{1}$ Emily Hohman, ${ }^{2}$ Jennifer S. Savage $\left(\mathbb{D},{ }^{2}\right.$ Daniel E. Rivera, ${ }^{3}$ \\ Penghong Guo, ${ }^{3}$ Krista S. Leonard, ${ }^{1}$ and Danielle Symons Downs $\mathbb{D}^{1,4}$ \\ ${ }^{1}$ Exercise Psychology Laboratory, Department of Kinesiology, The Pennsylvania State University, University Park, State College, \\ $P A, U S A$ \\ ${ }^{2}$ Center for Childhood Obesity Research, Department of Nutritional Sciences, \\ College of Health and Human Development, The Pennsylvania State University, University Park, PA, USA \\ ${ }^{3}$ School for Engineering of Matter, Transport, Energy, Arizona State University, Tempe, AZ, USA \\ ${ }^{4}$ Department of Obstetrics and Gynecology, College of Medicine, The Pennsylvania State University, Hershey, PA, USA
}

Correspondence should be addressed to Danielle Symons Downs; dsd11@psu.edu

Received 22 November 2017; Revised 21 June 2018; Accepted 26 July 2018; Published 1 October 2018

Academic Editor: Sharon Herring

Copyright (c) 2018 Abigail M. Pauley et al. This is an open access article distributed under the Creative Commons Attribution License, which permits unrestricted use, distribution, and reproduction in any medium, provided the original work is properly cited.

High gestational weight gain (GWG) in overweight/obese pregnant women increases maternal-fetal complications. We conducted a 6-week GWG intervention based on an energy balance model that includes theories of planned behavior (TPB) and selfregulation constructs to promote exercise and healthy eating motivation and behaviors. The purposes of this proof-of-concept feasibility study were to examine: (1) the energy balance model constructs over the intervention, and (2) pre-post intervention, weekly, and dose-response changes in study constructs. Methods. Overweight/obese pregnant women $(N=17)$ were randomized to 1 of 6 conditions, increasing in intensity, and included varied combinations of components (exercise sessions, healthy eating demonstrations, etc.). Exercise and healthy eating TPB (attitude, subjective norm, perceived behavioral control, intention), and self-regulation (prospective, retrospective) constructs were collected weekly. Exercise behavior, energy intake, and GWG were collected daily. Results. We observed: (a) significant increases in exercise TPB constructs, healthy eating attitude (limit unhealthy foods), exercise/healthy eating retrospective self-regulation; (b) significant decrease in healthy eating subjective norm (limit unhealthy foods); (c) trending increases for healthy eating perceived behavioral control (limit unhealthy foods), healthy eating prospective self-regulation, and energy intake; (d) significantly higher active time, steps, and energy expenditure at W3 relative to other weeks; (e) no significant increase in GWG; and, (f) a dose response effect such that women in more intensive dosages had greater gains in exercise and healthy eating perceived behavioral control (eat healthy/limit unhealthy foods). Conclusion. Brief exposure to a theoretically-driven, GWG intervention resulted in changes to exercise and healthy eating TPB and self-regulation motivational determinants, no significant increase in GWG, and suggests intervention intensity can strengthen perceived ability to engage in exercise/healthy eating behaviors; offering initial proof-of-concept for the intervention to regulate GWG in overweight/obese pregnant women. Future research will test this intervention over the course of pregnancy to understand long-term impact on maternal-fetal health outcomes.

\section{Introduction}

Over half of all overweight and obese pregnant women gain weight in excess of the current gestational weight gain
(GWG) recommendations $[1,2]$. This is problematic because high GWG increases the risk for preterm delivery, gestational diabetes, vascular disease, hypertensive disorders of pregnancy, and postpartum weight retention [1]. Even more 
alarming, high GWG elevates the risk for fetal morbidity including macrosomia (i.e., birth weight $>4,000$ g regardless of gestational age), birth trauma (e.g., shoulder dystocia), and longer hospital stays $[1,3]$. Data from the National Vital Statistics System [2] indicate that a high percentage of overweight (61\%) and obese (55\%) women are at greater risk for exceeding the IOM GWG guidelines (i.e., total weight gain: 15-25 pounds for overweight; $11-20$ pounds for obese) and therefore warrant intervention to effectively manage GWG for optimal maternal and fetal health.

Of particular concern, the rate of overweight/obese pregnant women exceeding GWG guidelines is predicted to increase given the lack of a "gold standard" intervention or clinical treatment [4]. Furthermore, the majority of interventions that aimed to reduce GWG in overweight/obese pregnant women have either yielded minimal effects or have been unsuccessful altogether [5-9]. However, information can be learned from the few effective studies. Vesco et al. [10] conducted a group-based weight management intervention in obese pregnant women using an energy-reduced DASH diet, recommendation of 30 minutes of moderate physical activity, and individual and group education sessions. They observed lower GWG in the intervention group from baseline (7-21 weeks gestation) through follow-up (34 weeks gestation) compared to the control group. Sagedal et al. [11] conducted an intervention where pregnant women received dietary counseling and twice-weekly exercise classes. They observed lower mean GWG in the intervention group compared to controls who received routine prenatal care. Despite these findings, the majority of participants in both studies still exceeded the IOM GWG recommendations. Sagedal et al. [11] also reported the proportion of women who exceeded the IOM GWG guidelines did not differ between condition, suggesting that a more intensive intervention approach (e.g., individually tailored exercise and dietary guidance) addressing the unique needs of overweight/obese women is needed to effectively manage GWG $[4,11,12]$.

Given this premise, we developed an individually tailored GWG intervention to manage weight in overweight/obese pregnant women based on a model of energy balance that includes the theories of planned behavior (TPB) and selfregulation [13-19]. The TPB aims to explain behavior through underlying constructs of attitude (positive or negative evaluation of the behavior), subjective norm (perceived social support to engage in the behavior), and perceived behavioral control (ease or difficulty in performing the behavior), which influence intention (person's level of motivation) to perform or not perform a behavior such as exercise or healthy eating [20]. Self-regulation is the ability for a person to work towards a goal by monitoring and managing thoughts, feelings, and behaviors, which is critical to changing behaviors such as exercise and healthy eating [21, 22]. Targeting both the TPB constructs and self-regulation simultaneously within an intervention can strengthen the likelihood of changing behavior, especially when the intervention is individually tailored based on the participant's level of the construct, for example, using strategies such as daily self-monitoring, positive encouragement, and reinforcement for a woman with low perceived behavioral control for exercising and eating healthy.

Previous work by Symons Downs et al. [23] established the utility to increase exercise determinants in pregnant women with gestational diabetes, but there is little research that has examined the associations between TPB and selfregulation constructs among pregnant women within the context of a GWG management intervention, especially in overweight/obese pregnant women. Thus, we conducted a brief, 6-week trial of an intervention focusing on exercise and healthy eating motivation and behaviors to manage GWG in overweight/obese pregnant women $[18,19]$. The overall goal of this proof-of-concept trial was to establish feasibility of the intervention components and the extent to which the TPB and self-regulation constructs motivated overweight/obese pregnant women to engage in exercise and healthy eating behaviors (as exercise and healthy eating are essential for regulating GWG). The purposes of this feasibility study were to (1) descriptively examine the energy balance model constructs over the 6-week pilot intervention and (2) examine pre-post intervention, weekly, and dose-response changes in the study constructs. Since the intervention period was brief, and intervention behavior change often takes longer than 6 weeks, we anticipated to observe changes in the underlying TPB and self-regulation motivational determinants without our energy balance model rather than observing changes in behavior (exercise, healthy eating, and GWG) [24]. We also expected to observe a dose-response effect such that the TPB and self-regulation constructs would improve with an increase in intervention dosage intensity. Our future research will test this GWG intervention in a larger trial over the course of pregnancy to understand long-term changes in exercise, healthy eating, and GWG as well as impact on additional maternal-infant outcomes.

\section{Methods}

2.1. Participants. Overweight/obese pregnant women $(N=17 ; M$ age $=29.4$ years, $\mathrm{SD}= \pm 5.6)$ were recruited using on-site clinic (e.g., speaking with potential participants after a prenatal appointment) and community (e.g., ads, flyers, and word of mouth) methods in locations in Central Pennsylvania. Women were randomized to 1 of 6 intervention dosages, which increased in intensity, for 6 weeks using a statistician-developed scheme placing an equal distribution of overweight and obese women in each dosage (Figure 1). Inclusion criteria were first pregnancy (no prior full-term ( $\geq 37$ weeks gestation) births), between 12 and 28 weeks gestation, $18-45$ years old, body mass index $(\mathrm{BMI}) \geq 25$, English-speaking, and no contraindications to exercise or eating healthy foods $[25,26]$. Physician's consent was obtained from the women's obstetrics and gynecology providers prior to enrollment.

\subsection{Study Design}

2.2.1. Baseline and Follow-Up Assessments. Participants were met at the University's Clinical Research Center, explained study procedures, and obtained informed consent. 


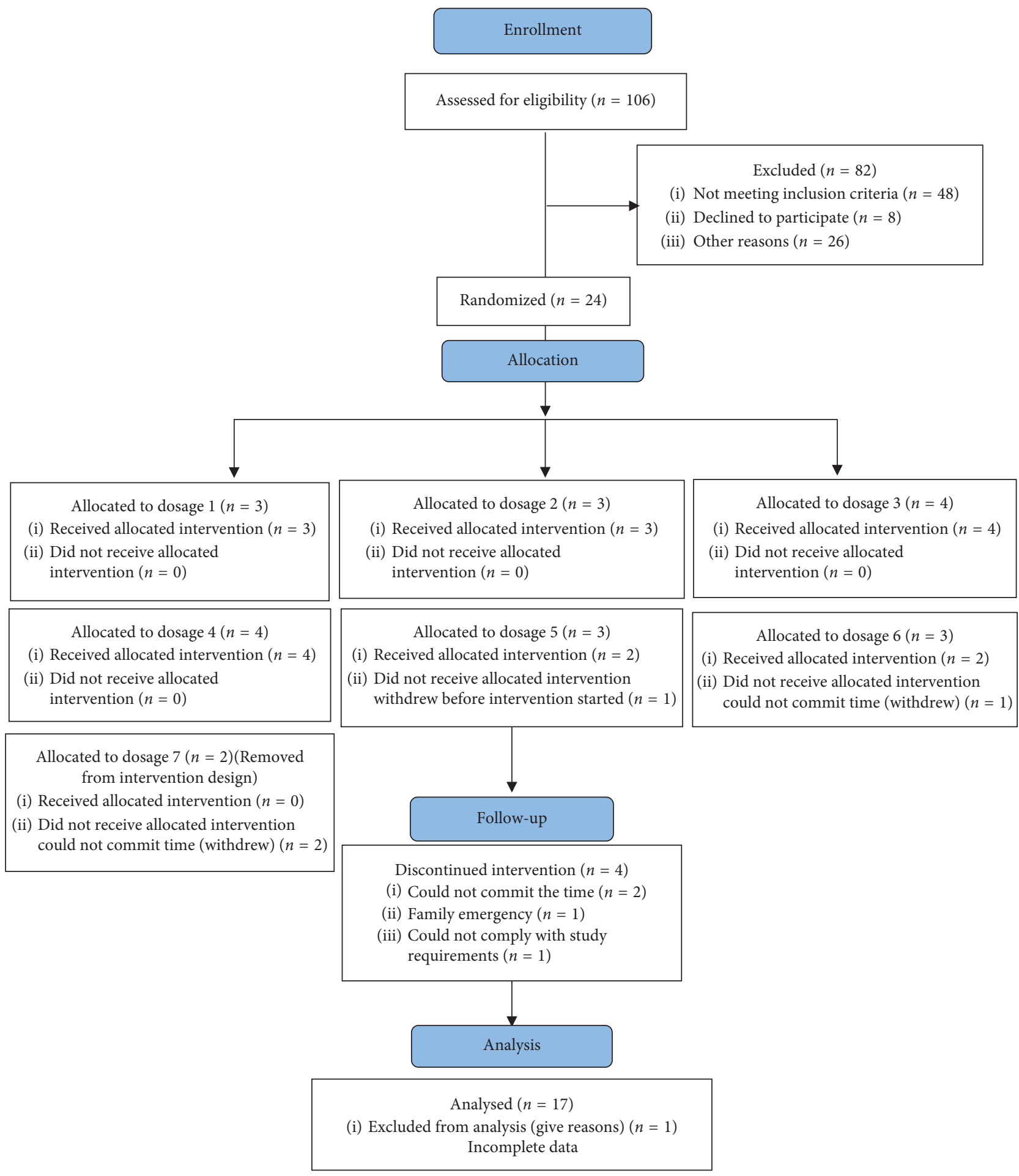

FIGURE 1: Consort recruitment table.

Eligible and interested women completed a brief medical exam to ensure safety for participation followed by measures of their height, weight, and blood pressure. During the baseline assessment, women also completed self-reported measures of exercise and healthy eating TPB (attitude, subjective norm, perceived behavioral control, and intention) and self-regulation (prospective and retrospective) constructs and personal demographics (e.g., age and BMI).
Women were given instructions on how to complete selfreported measures using an online data capture system (REDCap; [27]). Women were given a wrist-worn exercise activity monitor (to measure active time, energy expenditure in kcal, and steps), Wi-Fi weight scale, and instructions on how to use these devices as well as how to complete diet records using a smartphone app for three days during baseline. During the intervention, women completed weekly 
measures of the exercise and healthy eating TPB and selfregulation constructs and daily measures exercise behavior (wrist-worn activity monitor) and weight (Wi-Fi scale); energy intake was assessed three days/week using the smartphone app for dietary record intake, but for the current analyses, a back-calculation method was used for energy intake (described below). The same procedures (not including the medical exam and technology trainings) were used to collect data at the follow-up assessment.

2.2.2. Intervention. Intervention dosages were based on principles of behavior change, TPB, and self-regulation $[20,28,29]$, content from past successful interventions $[23,30]$, and initial prototype development feedback from pregnant women. Intervention dosages were built upon one another in a "step-up" design for intensity such that Dosage 1 included the "baseline intervention" with education on principles of exercise, healthy eating, GWG, goal-setting, and self-monitoring. Dosage 2 included the baseline intervention (i.e., Dosage 1) + a "step-up" of 30 min of healthy eating active learning (e.g., cooking demonstrations, recipe preparation, understanding principles of portion size, and energy density). The dosages increased in intensity with added exercise sessions, self-monitoring with instructor feedback, and healthy eating meal replacements up through Dosage 6. We originally designed the intervention with seven dosages; however, feedback from women during prototype development found this dosage to be too intensive, so we removed it from the study design. Figure 2 illustrates the six intervention dosages that were tested in this study.

2.3. Measures. Study measures of exercise and healthy eating TPB and self-regulation were collected weekly for six weeks (i.e., week $1=$ baseline, weeks $2-5$ of intervention, and week $6=$ follow-up) via online surveys. Intensive longitudinal data inform interventions and efficacy by capturing people's lives while they are actually living [31] which is a different approach from conventional experimental/survey research. All TPB constructs were measured on a 7-point Likert scale with higher scores indicating more positive attitude, subjective norm, perceived behavioral control, and intention.

2.3.1. Exercise TPB Constructs. Attitude was assessed with 7 differential pairs (e.g., useless-useful) that described how women felt about exercising for at least 30 minutes/day of accumulated moderate physical activity on most, if not all, days of the week [32,33]. Subjective Norm was assessed with three items (e.g., strongly disagree-strongly agree) measuring perceived support from important others to exercise for 30 minutes/day on most days in the following week. Perceived Behavioral Control was assessed with 3 items (e.g., for me to be physically active each day in the next week will be: extremely difficult/very little control/strongly disagreeextremely easy/complete control/strongly agree). Intention to engage in 30 minutes of exercise on most days of the week was assessed with six items (e.g., I intend to be physically active each day in the next week: strongly disagree-strongly agree). Internal consistency scores for the exercise TPB measures ranged from alpha $=0.77-0.98$ from pre-post for all constructs. Compliance of the exercise TPB measures was $99 \%$.

2.3.2. Healthy Eating TPB Constructs. Authors developed the healthy eating TPB scale by modifying the validated exercise TPB items. Eating Attitudes were assessed with 14 differential pairs, 7 assessing healthy eating attitudes (how women felt about eating healthy foods each day in the next week) and 7 assessing attitudes about limiting unhealthy foods (i.e., sugary beverages, eating chips, candy, baked goods, and fried foods each day in the next week). Subjective Norm was assessed using six items; three items measured women's perceptions of the extent to which significant others (e.g., husband, mother, and friend) provided support for them to eat healthy each day in the next week and three items assessed perceived support from others to limit unhealthy foods. Perceived Behavioral Control was assessed using six items; three items measured the ease or difficulty in eating healthy foods each day in the next week and three items assessed the ease or difficulty in limiting unhealthy foods each day in the next week. Intention was assessed using 12 items (e.g., strongly disagree/definitely not/not at allstrongly agree/definitely/very much). Three items were adapted to healthy eating from the exercise intention measure. Three items asked about women's intention to limit unhealthy foods. Three items assessed women's intention/motivation/plan to eat healthy and three items assessed intention to limit unhealthy foods each day in the next week. Internal consistency scores for the healthy eating and limiting unhealthy food TPB measures ranged from alpha $=0.73-0.98$ from pre-post for all constructs. Compliance of both, the healthy eating and limiting unhealthy food TPB measures, was $99 \%$.

2.3.3. Exercise Self-Regulation. Exercise self-regulation was assessed with 16 items to evaluate the degree to which women self-regulated their exercise behavior (e.g., if I achieve my short-term (daily or weekly) goals for exercise, I will feel proud; [28, 29, 34]). Eight items assessed prospective (i.e., in the next week) and eight items assessed retrospective (i.e., in the past week) self-regulation. Items were divided into 7 subscales: self-monitoring, goal-setting, action planning, coping planning, scheduling, cuing, and affective reaction. Internal consistency scores were excellent (alpha $=0.92-0.97)$ across time points. Compliance of the exercise self-regulation measure was $99 \%$.

2.3.4. Healthy Eating Self-Regulation. Healthy eating selfregulation was assessed with 16 items to evaluate the degree to which women self-regulates their healthy eating behavior (e.g., if I achieve my short-term (daily or weekly) goals for eating healthy, I will feel proud; $[28,29])$. Eight items assessed prospective and eight items assessed retrospective healthy eating self-regulation. Items were divided into 7 


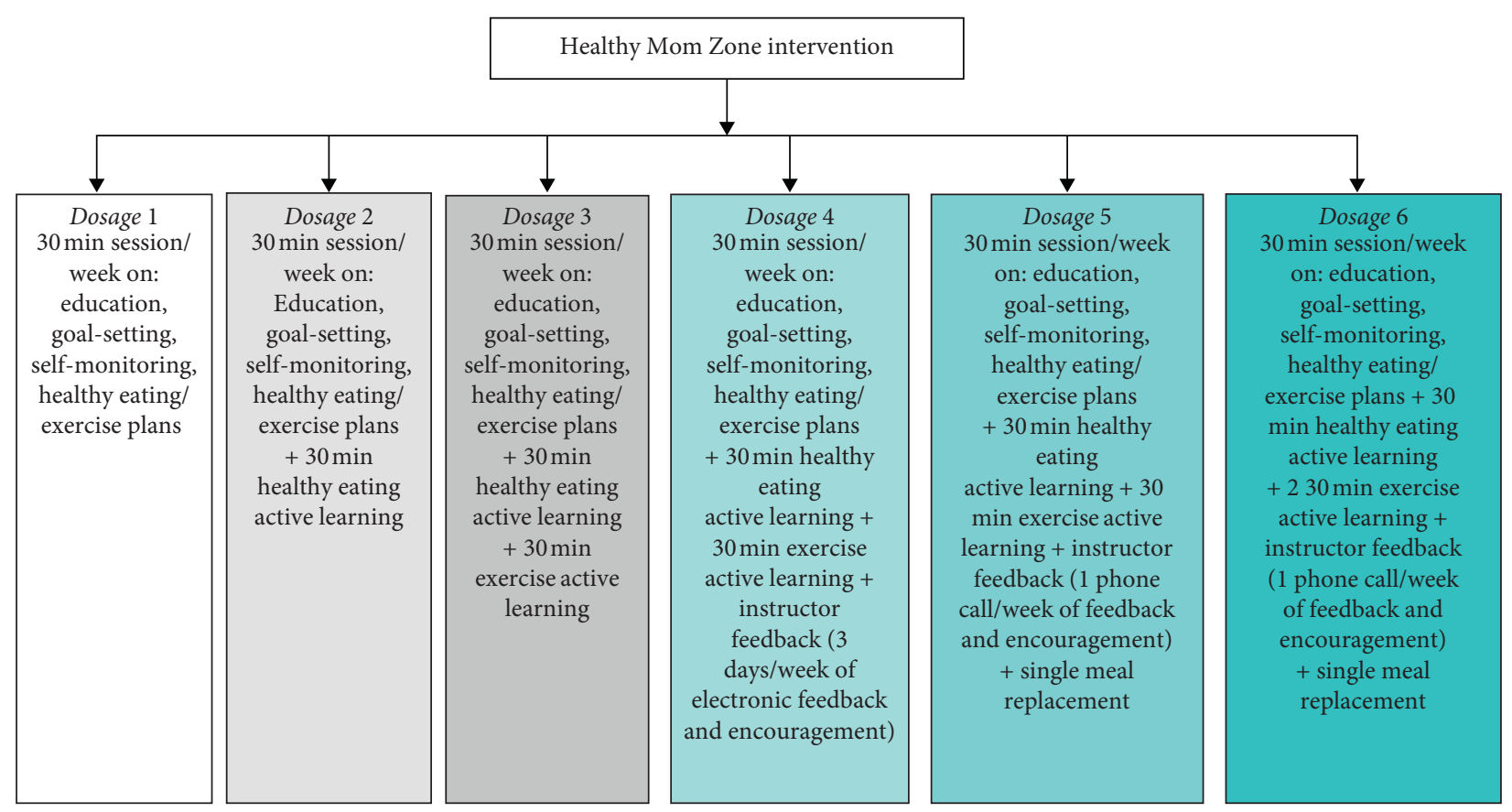

FIgURE 2: Healthy mom zone intervention dosages description.

subscales: self-monitoring, goal-setting, action planning, coping planning, scheduling, cuing, and affective reaction. Internal consistency scores ranged from alpha $=0.83-0.93$ across the time points. Compliance of the healthy eating selfregulation measure was $99 \%$.

\subsubsection{Exercise Behavior. Exercise behavior was assessed} using a wrist-worn activity monitor to measure daily activity time, energy expenditure, and steps $[35,36]$. Women wore the activity monitor 24 hours/day over the entire intervention period. Each woman had her own wrist monitor that connected to her phone via a mobile app to use as a selfmonitoring tool. Compliance with the monitors was $93 \%$.

2.3.6. Energy Intake. Energy intake was assessed using a back-calculation equation of energy intake [16] to address concerns about under/overreporting of energy intake when using self-report (pencil and paper or smartphone app) food records [37]. In the calculation, the participant's weight and energy expenditure are used to predict energy intake. Energy intake was predicted for each day over the 6-week intervention using average $\mathrm{kcal} / \mathrm{day}$.

2.3.7. GWG. GWG was assessed daily using a Wi-Fi scale over the 6-week study period. Women weighed themselves each day as soon as they woke up. The scale transmitted weights automatically to secure participant online accounts; online data were accessed and stored in REDCap. Compliance with the scale was $87 \%$.

2.4. Data Analysis. Descriptive statistics were used to examine study means, standard deviations, and frequencies.
Changes in TPB and self-regulation scores from baseline to follow-up were calculated with $t$-tests. Repeated-measures ANCOVA controlling for gestational age, dosage (1-6), and prepregnancy BMI was conducted to examine the weekly changes in the planned behavior/self-regulation constructs, exercise, energy intake, and GWG. Effects of study dosage on pre-post changes were assessed with ANOVA. Analyses were performed in SAS 9.4 (SAS Institute, Cary, NC) and SPSS 25 (IBM Corp., Armonk, New York). As this is a pilot study, the $p$ value for statistically significant differences overtime was set to $p \leq 0.05$ and the $p$ value for trends overtime was set to $p \leq 0.10$ [38].

\section{Results}

3.1. Demographics. The sample $(N=17)$ was homogenous; most participants were Caucasian, married, completed college, and had a family income of $\$ 40,000$ or higher per year ( $\mathrm{Ta}$ ble 1). Prepregnancy BMI of participants was in the overweight range $\left(M \mathrm{BMI}=29.1 \mathrm{~kg} / \mathrm{m}^{2}, \mathrm{SD}= \pm 3.8\right.$, range $=24.7-39 ; 65 \%$ overweight, $35 \%$ obese). Women were randomized to 1 of 6 dosages: Dosage $1 n=3$, Dosage $2 n=3$, Dosage $3 n=4$, Dosage $4 n=4$, Dosage $5 n=2$, and Dosage $6 n=2$.

\subsection{Pre-Post Intervention Change in Energy Balance Model} Constructs. There was a significant increase in healthy eating attitude (limit unhealthy foods, $p=0.046$ ) and a trend for an increase in healthy eating perceived behavioral control (limit unhealthy foods, $p=0.06$, Figure 3 ). There was a significant increase in retrospective exercise and healthy eating selfregulation $(p=0.004, p=0.0001)$. There were no significant changes for any of the other exercise/healthy eating TPB or self-regulation constructs, exercise and healthy eating behaviors, or GWG. 
TABle 1: Demographic characteristics of the study sample.

\begin{tabular}{|c|c|c|c|}
\hline \multirow{2}{*}{ Variable } & \multicolumn{3}{|c|}{ Total sample $N=17$} \\
\hline & M & $\mathrm{SD}$ & $\%$ \\
\hline Age & 29.4 & 5.6 & \\
\hline Body mass index (BMI) & 29.1 & 3.8 & \\
\hline Gestational age at study start & 16.4 & 4.5 & \\
\hline Weight at study start (pounds) & 185.74 & 27.49 & \\
\hline Weight gain over 4-week dosage (pounds) & 6.6 & 4.5 & \\
\hline \multicolumn{4}{|l|}{ Marital status } \\
\hline Married & & & 75.0 \\
\hline Not married living with partner & & & 18.75 \\
\hline Single & & & 6.25 \\
\hline Divorced & & & 0 \\
\hline \multicolumn{4}{|l|}{ Race } \\
\hline Caucasian & & & 94.5 \\
\hline Hispanic & & & 5.5 \\
\hline \multicolumn{4}{|l|}{ Education } \\
\hline Graduate & & & 23.5 \\
\hline College & & & 76.5 \\
\hline High school & & & 0 \\
\hline \multicolumn{4}{|l|}{ Family income } \\
\hline$\$ 100,000$ & & & 29.4 \\
\hline$\$ 40-\$ 100,000$ & & & 52.9 \\
\hline$\$ 20-\$ 40,000$ & & & 11.7 \\
\hline$\$ 10-\$ 20,000$ & & & 5.9 \\
\hline
\end{tabular}

$M=$ mean; $\mathrm{SD}=$ standard deviation.

\subsection{Weekly Change in Energy Balance Model Constructs}

3.3.1. Exercise $T P B$. The repeated-measures model for exercise attitude was significant, Wilks lambda $=0.258$, $F=4.613, \quad p=0.028$. Overall, attitude increased from W1-W6 of the intervention with significant differences at W2-W4 $(p=0.044)$ and trending differences at W2-W6 $(p=0.078)$. The repeated-measures model for exercise subjective norm was also significant, Wilks lambda $=0.271$, $F=4.301, p=0.034$. Subjective norm increased from W1W6; however, these differences did not reach statistical significance. The repeated-measures models for exercise perceived behavioral control and intention were not significant, Wilks lambda $=0.900, F=0.178, p=0.963$, and Wilks lambda $=0.537, F=1.379, p=0.326$, respectively. However, exercise perceived behavioral control and intention had significant differences at W2-W5 ( $p=0.014, p=0.017$, respectively); perceived behavioral control also had trending differences at W2-W6 ( $p=0.070$; Table 2).

3.3.2. Healthy Eating ТРB. The repeated-measures model for healthy eating attitude, subjective norm, perceived behavioral control, and intention was not significant, Wilks lambda $=0.487, F=1.687, p=0.243$, Wilks lambda $=0.728$, $F=0.523, \quad p=0.753$, Wilks lambda $=0.786, \quad F=0.435$, $p=0.813$, and Wilks lambda $=0.388, F=2.522, p=0.118$, respectively. There were no significant changes across weeks. The repeated-measures models for healthy eating attitude, perceived behavioral control, and intention (limit unhealthy foods) were not significant, Wilks lambda $=0.493, F=1.646$, $p=0.253$, Wilks lambda $=0.553, F=0.969, p=0.503$, and

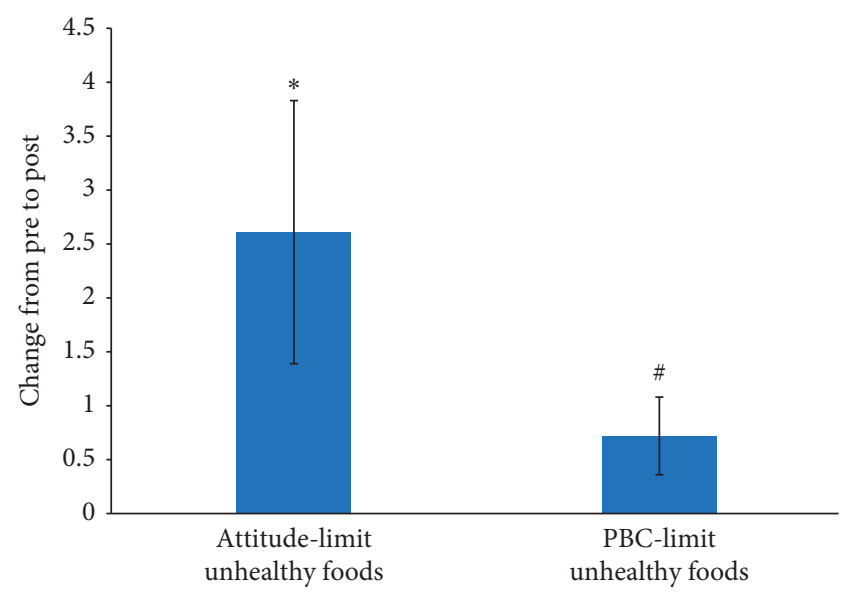

Figure 3: Change in TPB limit unhealthy eating constructs from pre- to post-assessment. Values are mean $\pm \mathrm{SE},{ }^{*} p<0.05,{ }^{\#} p<0.10$.

Wilks lambda $=0.631, F=0.934, p=0.507$, respectively. There were no significant changes across weeks. The repeated-measures model for healthy eating subjective norm (limit unhealthy foods) was significant, Wilks lambda $=0.094, F=9.659, p=0.013$. Subjective norm decreased from W1-W6; however, these differences did not reach statistical significance (Table 2).

3.3.3. Exercise and Healthy Eating Self-Regulation. The repeated-measures model for exercise self-regulation prospective was not significant, Wilks lambda $=0.744, F=0.549$, $p=0.736$; there were no significant differences across weeks. The repeated-measures model for exercise self-regulation retrospective was also not significant, Wilks lambda $=0.566, F=1.227, p=0.379$. However, significant weekly increases were observed from W2-W4, W2-W5, and W2-W6 ( $p=0.028,0.023,0.043$, respectively). The repeatedmeasures model for healthy eating self-regulation prospective trended toward significance, Wilks lambda $=0.358$, $F=2.873, p=0.089$. Healthy eating self-regulation prospective increased from W1-W6; however, these differences were not statistically significant. The repeated-measures model for healthy eating self-regulation retrospective was not significant, Wilks lambda $=0.542, F=1.350, p=0.335$. However, significant week increases were observed from W1-W3, W1-W4, W1-W6, and W2-W4 ( $p=0.017,0.014$, $0.016,0.043$, respectively) and a trending increase was observed at W1-W5 $(p=0.079)$ (Table 3).

3.3.4. Exercise and Healthy Eating Behavior. The repeatedmeasures model for active time was not significant, Wilks lambda $=0.494, F=1.024, p=0.490$; however, there were significant differences at $\mathrm{W} 2-\mathrm{W} 3, \mathrm{~W} 3-\mathrm{W} 4$, and $\mathrm{W} 3-\mathrm{W} 6$ ( $p=0.007, p<0.001, p<0.001$, respectively) and trending differences at W3-W5 $(p=0.086)$ such that W3 had significantly higher active time (e.g., $96 \mathrm{~min}$ ) compared to the other weeks (e.g., $72 \mathrm{~min}$ on average). The repeatedmeasures models for step and active kcal were also not 
TABLE 2: Exercise and healthy eating theory of planned behavior repeated measures.

\begin{tabular}{|c|c|c|c|c|c|c|c|c|c|c|c|c|c|c|c|c|}
\hline & \multicolumn{2}{|c|}{ Week 1} & \multicolumn{2}{|c|}{ Week 2} & \multicolumn{2}{|c|}{ Week 3} & \multicolumn{2}{|c|}{ Week 4} & \multicolumn{2}{|c|}{ Week 5} & \multicolumn{2}{|c|}{ Week 6} & \multirow{2}{*}{$\begin{array}{l}\text { Wilks } \\
\text { lambda }\end{array}$} & \multirow{2}{*}{$F$} & \multirow{2}{*}{$\begin{array}{c}\text { Partial } \\
\text { eta } \\
\text { squared }\end{array}$} & \multirow{2}{*}{$\begin{array}{c}p \\
\text { value }\end{array}$} \\
\hline & $M$ & SD & $M$ & SD & $M$ & SD & $M$ & SD & $M$ & SD & $M$ & SD & & & & \\
\hline $\begin{array}{l}\text { TPB exercise attitude } \\
(N=16)\end{array}$ & 6.08 & 0.83 & 6.09 & 0.67 & 6.31 & 0.60 & 6.32 & 0.68 & 6.22 & 0.70 & 6.32 & 0.79 & 0.258 & 4.613 & 0.742 & $0.028^{*}$ \\
\hline $\begin{array}{l}\text { TPB exercise subjective norm } \\
(N=16)\end{array}$ & 5.75 & 1.24 & 5.77 & 1.12 & 5.88 & 1.39 & 6.04 & 1.24 & 5.9 & 1.17 & 5.96 & 1.19 & 0.271 & 4.301 & 0.729 & $0.034^{*}$ \\
\hline $\begin{array}{l}\text { TPB exercise perceived } \\
\text { behavioral control }(N=16)\end{array}$ & 5.27 & 1.04 & 4.29 & 0.89 & 5.35 & 1.04 & 5.17 & 1.16 & 5.56 & 0.98 & 5.38 & 0.97 & 0.900 & 0.178 & 0.100 & 0.963 \\
\hline $\begin{array}{l}\text { TPB exercise intention } \\
(N=16)\end{array}$ & 5.41 & 1.39 & 5.23 & 0.97 & 5.58 & 1.00 & 5.33 & 1.1 & 5.67 & 0.99 & 5.44 & 1.21 & 0.537 & 1.379 & 0.463 & 0.326 \\
\hline $\begin{array}{l}\text { TPB healthy eating attitude } \\
(N=16)\end{array}$ & 6.30 & 0.695 & 6.30 & 0.41 & 6.37 & 0.55 & 6.36 & 0.57 & 6.40 & 0.64 & 6.47 & 0.59 & 0.487 & 1.687 & 0.513 & 0.243 \\
\hline $\begin{array}{l}\text { TPB healthy eating subjective } \\
\text { norm }(N=15)\end{array}$ & 6.29 & 0.93 & 6.18 & 1.10 & 6.04 & 1.11 & 6.16 & 1.17 & 6.02 & 1.23 & 6.04 & 1.27 & 0.728 & 0.523 & 0.272 & 0.753 \\
\hline $\begin{array}{l}\text { TPB healthy eating perceived } \\
\text { behavioral control }(N=16)\end{array}$ & 5.75 & 0.68 & 6.79 & 0.75 & 5.85 & 0.69 & 5.81 & 1.2 & 5.92 & 0.63 & 6.02 & 0.75 & 0.786 & 0.435 & 0.214 & 0.813 \\
\hline $\begin{array}{l}\text { TPB healthy eating intention } \\
(N=16)\end{array}$ & 5.92 & 0.98 & 6.05 & 0.74 & 6.07 & 0.83 & 5.93 & 0.87 & 6.01 & 0.86 & 6.13 & 0.93 & 0.388 & 2.522 & 0.612 & 0.118 \\
\hline $\begin{array}{l}\text { TPB limit healthy eating } \\
\text { attitude }(N=16)\end{array}$ & 5.92 & 0.85 & 6.19 & 0.58 & 6.21 & 0.6 & 6.20 & 0.65 & 6.19 & 0.68 & 6.32 & 0.64 & 0.493 & 1.646 & 0.507 & 0.253 \\
\hline $\begin{array}{l}\text { TPB limit healthy eating } \\
\text { subjective norm }(N=13)\end{array}$ & 6.41 & 0.81 & 6.23 & 0.92 & 6.05 & 1.10 & 6.15 & 1.18 & 6.10 & 0.81 & 6.00 & 1.34 & 0.094 & 9.659 & 0.906 & $0.013^{*}$ \\
\hline $\begin{array}{l}\text { TPB limit healthy eating } \\
\text { perceived behavioral control } \\
(N=14)\end{array}$ & 5.64 & 0.86 & 5.98 & 0.83 & 5.83 & 0.87 & 5.62 & 1.13 & 5.74 & 0.83 & 5.88 & 0.82 & 0.553 & 0.969 & 0.447 & 0.503 \\
\hline $\begin{array}{l}\text { TPB limit healthy eating } \\
\text { intention }(N=16)\end{array}$ & 5.56 & 1.28 & 5.78 & 1.03 & 5.74 & 0.91 & 5.65 & 0.89 & 5.73 & 0.96 & 5.85 & 0.95 & 0.631 & 0.934 & 0.369 & 0.507 \\
\hline
\end{tabular}

significant, Wilks lambda $=0.877, F=0.169, p=0.965$, and Wilks lambda $=0.841, F=0.189, p=0.954$, respectively. However, steps and active kcal at W3 were significantly higher than at W6 ( $p=0.013, p=0.006$, respectively). The repeated-measures model for energy intake trended toward significance, Wilks lambda $=0.003, F=73.617, p=0.088$. Overall, energy intake increased from W1-W6 of the intervention by $197 \mathrm{kcal}$; however, these weekly differences were not statistically significant (Table 4 ).

3.3.5. GWG. The repeated-measures model for GWG was not significant, Wilks lambda $=0.521, F=1.150, p=0.430$, and there were no significant changes across weeks (Table 5). GWG did not significantly increase over the 6-week intervention; observation of mean GWG across the weeks showed a similar pattern of approximately 1.1 pound change/week over the study period.

3.4. Dose-Response Change in Energy Balance Model Constructs. There was a positive relationship between study dosage and pre-post change in exercise perceived behavioral control $(p=0.07$; Figure $4(c))$, healthy eating perceived behavioral control $(p=0.03$; Figure $4(a))$, healthy eating perceived behavioral control for limiting unhealthy foods $(p=0.03$; Figure $4(\mathrm{~b}))$, healthy eating intention $(p=0.04)$, and healthy eating intention for limiting unhealthy foods $(p=0.03)$. In other words, women randomized to receive dosages at higher intensities had greater intention to eat healthy/limit unhealthy foods and their perception of eating healthy/limiting unhealthy foods and exercising was more positive. There were no significant dose-response pre-post changes in exercise and healthy eating self-regulation, exercise and healthy eating behaviors, or GWG.

\section{Discussion}

The objectives of this feasibility study were to descriptively examine the energy balance model constructs over the 6-week pilot intervention and examine pre-post intervention, weekly, and dose-response change in the study constructs. Overall, we found that brief exposure to the theoretically driven, GWG intervention resulted in significant changes to some of the exercise and healthy eating TPB and self-regulation motivational determinants, an increase in exercise behaviors at W3 of the intervention, and no significant changes to energy intake or GWG, supporting the initial proof-of-concept of the intervention among overweight/obese pregnant women. We also observed a dose-response effect such that an increase in intervention dosage was associated with greater exercise and healthy eating perceived control. These findings are further discussed below.

In partial support of our assumption, the intervention resulted in changes to some of the TPB and self-regulation motivational determinants over the intervention period. More specifically, we observed significant increases in 
TABLE 3: Exercise and healthy eating self-regulation repeated measures.

\begin{tabular}{|c|c|c|c|c|c|c|c|c|c|c|c|c|c|c|c|c|}
\hline & \multicolumn{2}{|c|}{ Week 1} & \multicolumn{2}{|c|}{ Week 2} & \multicolumn{2}{|c|}{ Week 3} & \multicolumn{2}{|c|}{ Week 4} & \multicolumn{2}{|c|}{ Week 5} & \multicolumn{2}{|c|}{ Week 6} & \multirow{2}{*}{$\begin{array}{l}\text { Wilks } \\
\text { lambda }\end{array}$} & \multirow{2}{*}{$F$} & \multirow{2}{*}{$\begin{array}{c}\text { Partial } \\
\text { eta } \\
\text { squared }\end{array}$} & \multirow{2}{*}{$\begin{array}{c}p \\
\text { value }\end{array}$} \\
\hline & $M$ & $\mathrm{SD}$ & $M$ & $\mathrm{SD}$ & $M$ & SD & $M$ & $\mathrm{SD}$ & $M$ & SD & $M$ & $\mathrm{SD}$ & & & & \\
\hline $\begin{array}{l}\text { Exercise prospective } \\
(N=16)\end{array}$ & 40.63 & 6.69 & 37.55 & 6.28 & 35.88 & 6.66 & 37.19 & 8.71 & 38.06 & 8.66 & 36.5 & 8.85 & 0.744 & 0.549 & 0.265 & 0.736 \\
\hline $\begin{array}{l}\text { Exercise } \\
\text { retrospective } \\
(N=16)\end{array}$ & 28.00 & 9.32 & 30.81 & 8.78 & 33.97 & 7.63 & 35.49 & 10.54 & 36.35 & 9.71 & 36.04 & 10.47 & 0.566 & 1.227 & 0.434 & 0.379 \\
\hline $\begin{array}{l}\text { Healthy eating } \\
\text { prospective }(N=16)\end{array}$ & 39.33 & 8.05 & 36.56 & 6.23 & 36.19 & 7.19 & 38.21 & 7.15 & 37.85 & 8.51 & 38.38 & 8.1 & 0.358 & 2.873 & 0.642 & $0.089^{\#}$ \\
\hline $\begin{array}{l}\text { Healthy eating } \\
\text { retrospective } \\
(N=16)\end{array}$ & 25.87 & 9.70 & 31.25 & 9.45 & 34.03 & 7.13 & 36.63 & 8.24 & 34.56 & 9.83 & 37.13 & 9.25 & 0.542 & 1.350 & 0.458 & 0.335 \\
\hline
\end{tabular}

TABle 4: Exercise and healthy eating behavior repeated measures.

\begin{tabular}{|c|c|c|c|c|c|c|c|c|c|c|c|c|c|c|c|c|}
\hline & \multicolumn{2}{|c|}{ Week 1} & \multicolumn{2}{|c|}{ Week 2} & \multicolumn{2}{|c|}{ Week 3} & \multicolumn{2}{|c|}{ Week 4} & \multicolumn{2}{|c|}{ Week 5} & \multicolumn{2}{|c|}{ Week 6} & \multirow{2}{*}{$\begin{array}{l}\text { Wilks } \\
\text { lambda }\end{array}$} & \multirow{2}{*}{$F$} & \multirow{2}{*}{$\begin{array}{c}\text { Partial eta } \\
\text { squared }\end{array}$} & \multirow{2}{*}{$\begin{array}{c}p \\
\text { value }\end{array}$} \\
\hline & $M$ & SD & $M$ & SD & $M$ & SD & $M$ & SD & $M$ & SD & $M$ & SD & & & & \\
\hline $\begin{array}{l}\text { Active time } \\
(N=13)\end{array}$ & 75.05 & 23.1 & 71.27 & 27.76 & 96.38 & 34.60 & 73.95 & 31.16 & 65.68 & 30.27 & 71.72 & 29.04 & 0.494 & 1.024 & 0.506 & 0.490 \\
\hline Steps $(N=14)$ & 7674 & 2688 & 7917 & 3323 & 8582 & 2997 & 8235 & 3345 & 7205 & 3228 & 7042 & 2794 & 0.877 & 0.169 & 0.123 & 0.965 \\
\hline $\begin{array}{l}\text { Energy } \\
\text { expenditure } \\
(N=13)\end{array}$ & 467.2 & 202.2 & 447.4 & 236.5 & 507.4 & 218.3 & 497.9 & 251.3 & 405.3 & 234.4 & 411.7 & 212.8 & 0.841 & 0.189 & 0.159 & 0.954 \\
\hline $\begin{array}{l}\text { Energy intake } \\
(N=9)\end{array}$ & 2755 & 928 & 2780 & 475 & 2935 & 541 & 2732 & 668 & 2887 & 550 & 2952 & 593 & 0.003 & 73.62 & 0.997 & $0.088^{\#}$ \\
\hline
\end{tabular}

${ }^{*} p<0.05 ;{ }^{\#} p<0.10$.

TABLE 5: Change in GWG repeated measures.

\begin{tabular}{|c|c|c|c|c|c|c|c|c|c|c|c|c|c|c|}
\hline & \multicolumn{2}{|c|}{$\Delta \mathrm{W} 1-\mathrm{W} 2$} & \multicolumn{2}{|c|}{$\Delta \mathrm{W} 2-\mathrm{W} 3$} & \multicolumn{2}{|c|}{$\Delta \mathrm{W} 3-\mathrm{W} 4$} & \multicolumn{2}{|c|}{$\Delta \mathrm{W} 4-\mathrm{W} 5$} & \multicolumn{2}{|c|}{$\Delta \mathrm{W} 5-\mathrm{W} 6$} & \multirow{2}{*}{ Wilks lambda } & \multirow{2}{*}{$F$} & \multirow{2}{*}{ Partial eta squared } & \multirow{2}{*}{$p$ value } \\
\hline & $M$ & $\mathrm{SD}$ & $M$ & $\mathrm{SD}$ & $M$ & $\mathrm{SD}$ & $M$ & $\mathrm{SD}$ & $M$ & $\mathrm{SD}$ & & & & \\
\hline GWG $(N=12)$ & 1.50 & 1.17 & 0.65 & 1.48 & 1.08 & 0.94 & 1.00 & 0.87 & 1.59 & 0.85 & 0.521 & 1.150 & 0.479 & 0.430 \\
\hline
\end{tabular}

${ }^{*} p<0.05 ;{ }^{\#} p<0.10$. Weight is measured in pounds.

exercise attitude, subjective norm, perceived behavioral control, and intention, healthy eating attitude (limit unhealthy foods) and exercise/healthy eating retrospective selfregulation, a trend toward significance for an increase in healthy eating perceived behavioral control (limit unhealthy foods) and healthy eating prospective self-regulation. These findings are consistent with our past research among pregnant women with gestational diabetes [23], and they suggest that brief exposure to the intervention can positively impact how women feel about exercise and healthy eating.

These findings also illustrate a positive impact on women's perceived ability and self-regulation for exercise and healthy eating, which in turn can have a positive impact on these behaviors to help better regulate GWG. More specifically, the intervention education and skills (including exercise and healthy eating action plans) taught to the women along with the use of mHealth tools (e.g., Wi-Fi scale, wrist-worn activity monitor, and smartphone app for intake served as both intervention self-regulatory tools and behavioral measures) positively influenced women's retrospective and prospective self-regulation. These findings also suggest that women felt more comfortable with regulating their exercise and eating behaviors from week to week and were able to think about setting goals and action plans in advance of an upcoming week. These findings are promising for the future intervention that will require women to selfregulate exercise and healthy eating behaviors over the duration of pregnancy to regulate their weight gain. While we did not find significant pre-post intervention change in exercise perceived behavioral control and intention, there were significant increases in exercise perceived behavioral control and intention from $\mathrm{W} 2-\mathrm{W} 5$ and a trend toward significance for exercise perceived behavioral control from W2-W6. This suggests that the accumulation of the education and skills learned over the course of the short intervention period provided the women with tools to manage perceived barriers and improve their intention for exercise. These weekly findings are promising for the design of the future intervention as they may be replicated over a longer intervention period. Similarly, with a trend toward 


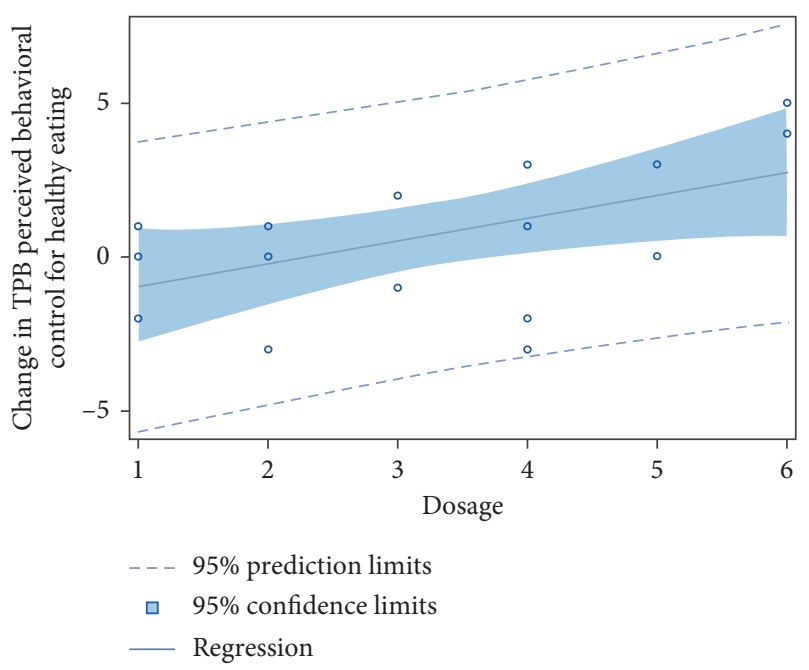

(a)

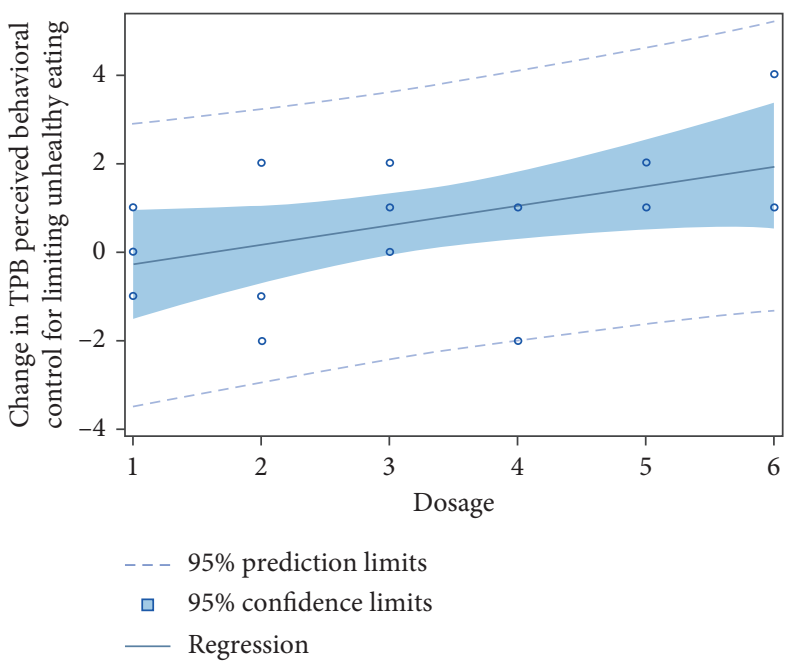

(b)

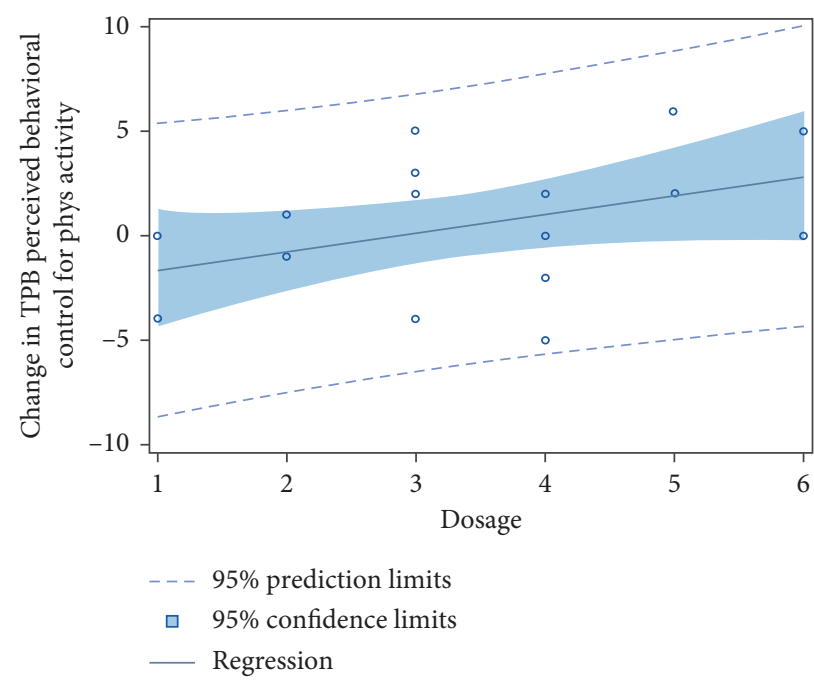

(c)

FIGURE 4: Intervention dose was positively associated with pre-post change in perceived behavioral control for (a) healthy eating ( $p=0.03)$, (b) limiting unhealthy eating $(p=0.03)$, and (c) exercise $(p=0.07)$.

significance for perceived behavioral control (limit unhealthy foods), the program content appears to provide women with useful strategies to overcome key barriers to eating healthy foods and improved their perceived ability to reduce consumption of unhealthy foods. The positive findings for increases in exercise perceived behavioral control and healthy eating perceived behavioral control (limit unhealthy foods) are particularly important for designing interventions as perceived behavioral control is a strong determinant of behavior in pregnant women [32] and will play a key role in the future intervention to manage GWG over the entire course of pregnancy.

Moreover, while we found increases in exercise subjective norm and healthy eating attitude (limit unhealthy foods), we interestingly observed a significant decrease in healthy eating subjective norm (limit unhealthy foods) over the intervention period. One explanation for these findings may be that women are exposed to intervention content on strategies to exercise and eat healthy, and this content partly focuses on the role that friends and family have in supporting these behaviors. While the women themselves may have improved their own feelings about the benefits of exercise and eating healthy through the intervention exposure, it is possible that some of the women also gained insight to barriers in their support system to eating healthy (e.g., husband or family poor eating habits) that decreased their subjective norm for limiting unhealthy foods. Future research is needed to better understand these normative influences for limiting unhealthy foods; additional intervention content on social influences may be needed in the future larger intervention to help women overcome these barriers.

Although we did not assume that brief exposure of the intervention would result in significant changes to exercise and healthy eating behaviors, we nevertheless observed some interesting findings. For example, although exercise activity 
time at W6 of the intervention was slightly lower (71 min) compared to $\mathrm{W} 1$ (75 $\mathrm{min})$, exercise activity time was considerably higher at W3 (96 min) compared to all other weeks (e.g., range 65-75 $\mathrm{min}$ ), and there was a significant increase in time from W2-W3. A similar pattern was observed for activity steps and energy expenditure kcal with the highest observed values at W3 of the intervention. It is possible that women started to feel more comfortable with the intervention by $\mathrm{W} 3$, and this resulted in a spike in their activity, but then they may have encountered some barriers (e.g., soreness or fatigue from exercise; thinking they were doing "too much too fast") that led to a decline in activity following W3. Even though the exercise sessions recommend activity within the guidelines $(150 \mathrm{~min} /$ week of moderate intensity aerobic activity $[25,26])$, the recommendation may have been perceived to be more intense by some than it actually was. Given that intervention exposure longer than six weeks is generally needed to see impact on behavioral changes [24], and we did not obtain weekly feedback on the women regarding specific exercise barriers, some caution is warranted with interpreting these findings. However, considering these findings in combination with the promising evidence for the positive effects of the intervention on the exercise motivational determinants, there is proof-of-concept for the intervention impacting exercise behaviors. Future research is needed to test this assumption over a longer period of time during pregnancy to understand changes in exercise as a result of the intervention.

Moreover, while energy intake trended toward a significant increase over the study period, it is important to note that (a) pregnant women are supposed to increase their energy intake in pregnancy and adjust it relative to their activity level [39], and (b) the observed increase from W1 to W6 was only a difference of approximately $200 \mathrm{kcal}$. In other words, there is proof-of-concept for the intervention to regulate energy intake such that it did not lead to a significant increase in kcal over the study period. Also, caloric needs for pregnant women differ across the trimesters, so the increase in kcal could be related to the range in gestational age of the participants [39] (e.g., gestational age at study start ranged from 12 to 25 weeks and at study end, ranged from 18 to 31 weeks). Increases in caloric needs over the course of pregnancy need to be taken into consideration when developing interventions to manage GWG. Future research is needed to understand the impact of our intervention on energy intake over the entire pregnancy and in relation to GWG. Furthermore, although we did not anticipate to find any significant differences in GWG, it is nevertheless important to note that we did not observe a significant increase in GWG over the course of the intervention period. The average change in GWG from week to week was $\sim 1.1$ pounds. Although this rate of change is higher than is recommended for overweight/obese pregnant women (e.g., average range of $0.4-0.7$ pounds per week after the 1 st trimester for overweight and obese, respectively; [1]), it is promising that we did not observe any large spikes in weight gain for most of the women. Future research is needed to test our intervention over the entire pregnancy to fully understand the impact on GWG.
Also, in partial support of our assumption, we observed a dose-response pre-post change in exercise perceived behavioral control, healthy eating perceived behavioral control, healthy eating perceived behavioral control (limit unhealthy foods), healthy eating intention, and healthy eating intention (limit unhealthy foods) but not exercise or healthy eating attitude or subjective norm. The doseresponse change in exercise perceived behavioral control is important to highlight because with the higher dosage, the woman's perceived ease to exercise increases as they are provided with the tools to perform the behavior and know it is safe. With the feeling of increased ease to exercise, women are more likely to continue with exercise, which in turn will help manage energy balance for healthy weight gain throughout pregnancy. Similarly, the dose-response in perceived behavioral control healthy eating and healthy eating (limit unhealthy food) suggests that with a higher intervention dosage, there is greater perceived ease of eating healthy and limiting unhealthy foods. Higher intervention dosages included more intensive education materials, greater number of cooking demonstrations, and meal replacements, which provided women with the perceived resources and skills to make it easier to eat healthy and limit unhealthy foods. Also, in higher dosages, women had greater intention to eat healthy foods and to limit unhealthy foods. Coupled with the positive increase in attitude to limit unhealthy foods from pre- to post-intervention, and the trend for an increase in perceived behavioral control to limit unhealthy foods, these findings illustrate proof-of-concept for our intervention dosages. This is particularly important as our future intervention trial will be adapted over the entire pregnancy to help women adjust to their individual needs. For example, if a woman is successfully regulating her weight gain and staying within her GWG goals, she will continue with the baseline intervention (e.g., similar to Dosage 1 in this study). However, if another woman is struggling with regulating her GWG within her goals, the intervention will be adapted (i.e., made more intensive by increasing the dosage as illustrated in dosages 2-6 in this study) in 3- to 4week cycles to manage GWG [19].

\section{Implications for Practice and/or Policy}

These study findings are important for clinical practice as they illustrate the importance of targeting motivational determinants for behavior change in addition to exercise and healthy eating to regulate GWG. Clinicians may want to consider talking with their overweight/obese prenatal patients more specifically about their attitudes, feelings, and barriers to making behavioral changes to better regulate their weight gain. Strengths of this study include the theoretically driven basis of the intervention, focus on overweight/obese women, population in need of managing GWG for healthy maternal and infant outcomes, randomized study design, and impact of the intervention on several outcomes after brief exposure. This study is also one of the first interventions to target both $\mathrm{TPB}$ and self-regulation constructs in an energy balance model for regulating GWG in overweight/obese pregnant women. These strengths are 
important for developing future interventions as well as disseminating information when helping pregnant women to regulate their GWG.

Limitations of this study include a small sample size with a homogenous sample (e.g., married, middle to upper class, Caucasian), which limits generalizability. Future research will aim to broaden generalizability of our study findings. Also, given the short duration of the intervention, we did not anticipate to see significant behavioral changes in exercise, energy intake, or GWG. Future research will need to examine the impact of the intervention on these behavioral targets over the full duration of pregnancy. Furthermore, although the healthy eating TPB measures were adapted from the validated exercise TPB measures, future research is needed to validate these items within our energy balance model.

\section{Conclusions}

Brief exposure to a theoretically driven, GWG intervention based on a model of energy balance resulted in significant increases to exercise and healthy eating motivational determinants, an increase in exercise in the middle of the intervention, and no significant changes to energy intake or GWG, supporting the initial proof-of-concept of the intervention among overweight/obese pregnant women. Future research will test this intervention in a larger trial over the entire duration of pregnancy to understand its impact on regulating GWG and maternal-infant health.

\section{Disclosure}

A poster related to this manuscript was presented at Obesity Week on November 11, 2016 (An Individually Tailored Gestational Weight Gain Intervention Impacts Determinants of Healthy Eating and Exercise Behaviors in Overweight and Obese Pregnant Women). The content is solely responsibility of the authors and does not necessarily represent the official views of the NIH.

\section{Conflicts of Interest}

The authors declare that they have no conflicts of interest.

\section{Acknowledgments}

The authors would like to acknowledge the assistance of the Healthy Mom Zone team who assisted with participant recruitment and data collection for this study. The project described was supported by the National Center for Advancing Translational Sciences and National Institutes of Health, through Grants UL1 TR000127 and TR002014. Support for this work has been provided by the National Heart, Lung, and Blood Institute (NHLBI) of the National Institutes of Health through Grant 1 R01 HL119245-01.

\section{References}

[1] Institute of Medicine, Weight Gain during Pregnancy: Reexamining the Guidelines, National Academies Press, Washington, DC, USA, 2009.
[2] National Vital Statistics System, "QuickStats: gestational weight gain among women with full-term, singleton births, compared with recommendations-48 States and the District of Columbia," Morbidity and Mortality Weekly Report, vol. 65, no. 40, p. 1121, 2016.

[3] J. M. Vernini, J. B. Moreli, C. G. Magalas, R. A. Cosat, M. V. Rudge, and I. M. Calderon, "Maternal and fetal outcomes in pregnancies complicated by overweight and obesity," Reproductive Health, vol. 13, no. 1, p. 100, 2016.

[4] D. S. Downs, "Obesity in special populations," Primary Care: Clinics in Office Practice, vol. 43, no. 1, pp. 109-120, 2016.

[5] J. M. Dodd, D. Turnbull, A. J. McPhee et al., "Antenatal lifestyle advice for women who are overweight or obese: LIMIT randomised trial," BMJ, vol. 348, article g1285, 2014.

[6] I. Guelinckx, R. Devlieger, P. Mullie, and G. Vansant, "Effect of lifestyle intervention on dietary habits, physical activity, and gestational weight gain in obese pregnant women: a randomized controlled trial," American Journal of Clinical Nutrition, vol. 91, no. 2, pp. 373-380, 2010.

[7] M. Hawkins, M. Hosker, B. H. Marcus et al., "A pregnancy lifestyle intervention to prevent gestational diabetes risk factors in overweight Hispanic women: a feasibility randomized controlled trial," Diabetic Medicine, vol. 32, no. 1, pp. 108-115, 2015.

[8] R. A. Krukowski, D. S. West, M. DiCarlo, M. A. Cleves, M. E. Saylors, and A. Andres, "Association of gestational weight gain expectations and advice on actual weight gain," Obstetrics and Gynecology, vol. 129, no. 1, pp. 76-82, 2017.

[9] L. Poston, A. L. Briley, S. Barr et al., "Developing a complex intervention for diet and activity behaviour change in obese pregnant women (the UPBEAT trial); assessment of behavioural change and process evaluation in a pilot randomised controlled trial," BMC Pregnancy and Childbirth, vol. 13, p. 148, 2013.

[10] K. K. Vesco, N. Karanja, J. C. King et al., "Efficacy of a groupbased dietary intervention for limiting gestational weight gain among obese women: a randomized trial," Obesity, vol. 22, no. 9, pp. 1989-1996, 2014.

[11] L. Sagedal, N. Øverby, E. Bere et al., "Lifestyle intervention to limit gestational weight gain: the Norwegian fit for delivery randomised controlled trial," BJOG: An International Journal of Obstetrics and Gynaecology, vol. 124, no. 1, pp. 97-109, 2017.

[12] R. A. Krukowski, D. West, M. DiCarlo et al., "A behavioral intervention to reduce excessive gestational weight gain," Maternal and Child Health Journal, vol. 21, no. 3, pp. 485-491, 2016.

[13] Y. Dong, D. E. Rivera, D. M. Thomas et al., "A dynamical systems model for gestational weight gain behavioral interventions," in Proceedings of the 2012 American Control Conference, pp. 4059-4064, Montreal, QC, Canada, June 2012, PMID: 24309837.

[14] Y. Dong, D. E. Rivera, D. S. Downs, J. S. Savage, D. M. Thomas, and L. M. Collins, "Hybrid model predictive control for optimizing gestational weight gain behavioral interventions," in Proceedings of the 2013 American Control Conference, pp. 1970-1975, Washington, DC, USA, June 2013, PMID: 24336314, PMCID: PMC3856197.

[15] Y. Dong, S. Deshpande, D. E. Rivera, D. S. Downs, and J. S. Savage, "Hybrid model predictive control for sequential decision policies in adaptive behavioral interventions," in Proceedings of the 2014 American Control Conference, pp. 4198-4203, Portland, OR, USA, June 2014, ISBN: 978-1-47993271-9, PMID: 25635157, PMCID: PMC4307847. 
[16] P. Guo, D. E. Rivera, D. S. Downs et al., "Semi-physical identification and state estimation of energy intake for interventions to manage gestational weight gain," in Proceedings of the 2016 American Control Conference, pp. 1271-1276, Boston, MA, USA, July 2016, PMID: 27570366 PMCID: PMC5001697.

[17] J. S. Savage, D. S. Downs, Y Dong, D. E. Rivera, and L. C. Collins, "Control systems engineering for optimizing a prenatal weight gain intervention to regulate infant birth weight," American Journal of Public Health, vol. 104, no. 7, pp. 1247-1254, 2014.

[18] D. Symons Downs, J. S. Savage, D. E. Rivera et al., "Influence of a feasibility study on the design of an individually-tailored, adaptive intervention to manage weight in overweight and obese pregnant women," Annals of Behavioral Medicine, vol. 51, no. S1, pp. S1451-S1452, 2017.

[19] D. Symons Downs, J. S. Savage, D. E. Rivera et al., "Individually tailored, adaptive intervention to manage gestational weight gain: protocol for a randomized controlled trial in women with overweight and obesity," JMIR Research Protocols, vol. 7, no. 6, p. e150, 2018.

[20] I. Ajzen, "The theory of planned behavior," Organizational Behavior and Human Decision Processes, vol. 50, no. 2, pp. 179-211, 1991.

[21] C. S. Carver and M. F. Scheier, On the Self-Regulation of Behavior, Cambridge University Press, Cambridge, UK, 1998.

[22] H. Leventhal, D. Nerenz, and D. Steele, "Illness representations and coping with health threats," in A Handbook of Psychology and Health, A. Baum and J. Singer, Eds., vol. 4, pp. 219-252, Erlbaum, Hillsdale, NJ, USA, 1984.

[23] D. S. Downs, J. M. Dinallo, L. L. Birch, I. M. Paul, and J. S. Ulbrecht, "Randomized face-to-face vs. home exercise interventions in pregnant women with gestational diabetes," Psychology of Sport and Exercise, vol. 30, pp. 73-81, 2017.

[24] B. Fjeldsoe, M. Neuhaus, E. Winkler, and E. Eakin, "Systematic review of maintenance of behavior change following physical activity and dietary interventions," Health Psychology, vol. 30, no. 1, pp. 99-109, 2011.

[25] ACOG Committee on Obstetric Practice, "ACOG Committee opinion no. 267: exercise during pregnancy and the postpartum period," Obstetrics and Gynecology, vol. 99, no. 1, pp. 171-173, 2002.

[26] ACOG Committee on Obstetric Practice, "ACOG Committee opinion no. 650: physical activity and exercise during pregnancy and the postpartum period," Obstetrics and Gynecology, vol. 126, no. 6, pp. e135-e142, 2015.

[27] P. A. Harris, R. Taylor, R. Thielke, J. Payne, N. Gonzalez, and J. G. Conde, "Research electronic data capture (REDCap)a metadata-driven methodology and workflow process for providing translational research informatics support," Journal of Biomedical Informatics, vol. 42, no. 2, pp. 377-381, 2009.

[28] P. S. Petosa, Use of social cognitive theory to explain exercise behavior among adults, Ph.D. dissertation, The Ohio State University, Columbus, OH, USA, 1993.

[29] F. F. Sniehotta, R. Schwarzer, U. Scholz, and B. Schüz, "Action planning and coping planning for long-term lifestyle change: theory and assessment," European Journal of Social Psychology, vol. 35, no. 4, pp. 565-576, 2005.

[30] The Diabetes Prevention Program (DPP) Research Group, "The diabetes prevention program (DPP)," Diabetes Care, vol. 25, no. 12, pp. 2165-2171, 2002.

[31] T. A. Walls and J. L. Schafer, Models for Intensive Longitudinal Data, Oxford University Press, Oxford, UK, 2006.
[32] D. S. Downs and H. A. Hausenblas, "Exercising for two: Examining pregnant women's second trimester exercise intention and behavior using the framework of the theory of planned behavior," Women's Health Issues, vol. 13, no. 6, pp. 222-228, 2003.

[33] D. Symons Downs and H. A. Hausenblas, "Exercising during pregnancy and postpartum: an elicitation study using the framework of the theory of planned behavior," Journal of Midwifery and Women's Health, vol. 49, no. 2, pp. 138-144, 2004.

[34] M. R. Umstattd, R. Motl, S. Wilcox, R. Saunders, and M. Watford, "Measuring physical activity self-regulation strategies in older adults," Journal of Physical Activity and Health, vol. 6, no. S1, pp. S105-S112, 2009.

[35] T. Ferguson, A. V. Rowlands, T. Olds, and C. Maher, "The validity of consumer-level, activity monitors in healthy adults worn in free-living conditions: a cross-sectional study," International Journal of Behavioral Nutrition and Physical Activity, vol. 12, no. 1, pp. 1-9, 2015.

[36] F. A. Storm, B. W. Heller, and C. Mazzà, "Step detection and activity recognition accuracy of seven physical activity monitors," PLoS One, vol. 10, no. 3, Article ID e0118723, 2015.

[37] S. W. Lichtman, K. Pisarska, E. R. Berman et al., "Discrepancy between self reported and actual caloric intake and exercise in obese subjects," New England Journal of Medicine, vol. 327, no. 27, pp. 1893-1898, 1992.

[38] M. A. Hertzog, "Considerations in determining sample size for pilot studies," Research in Nursing \& Health, vol. 31, no. 2, pp. 180-191, 2008.

[39] M. A. Kominiarek and P. Rajan, "Nutrition recommendations in pregnancy and lactation," Medical Clinics of North America, vol. 100, no. 6, pp. 1199-1215, 2016. 


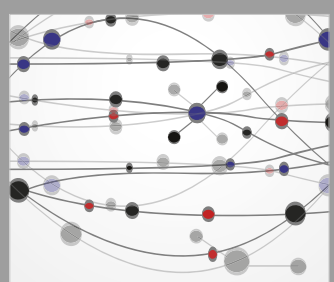

The Scientific World Journal
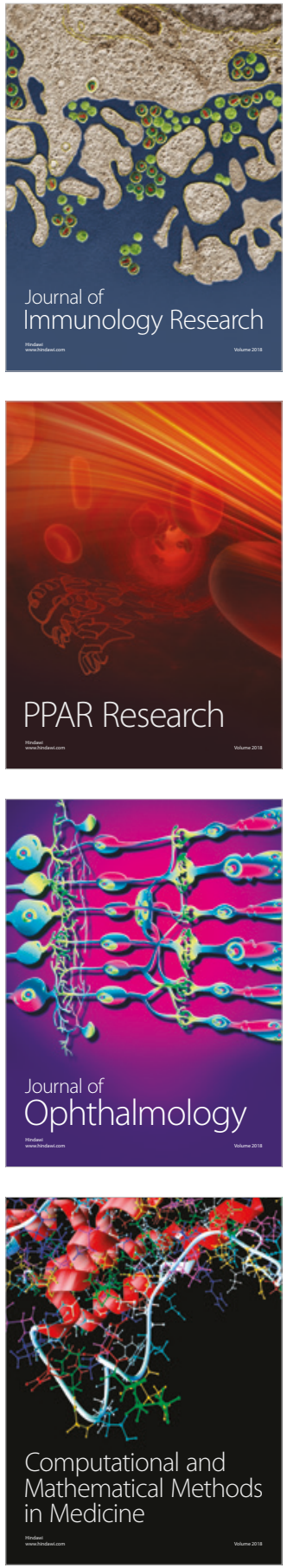

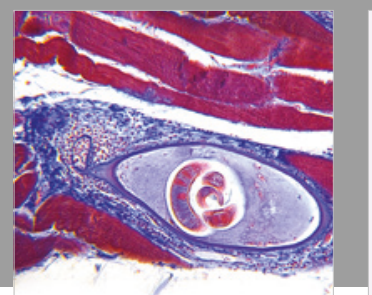

Gastroenterology Research and Practice

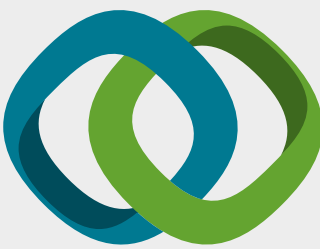

\section{Hindawi}

Submit your manuscripts at

www.hindawi.com
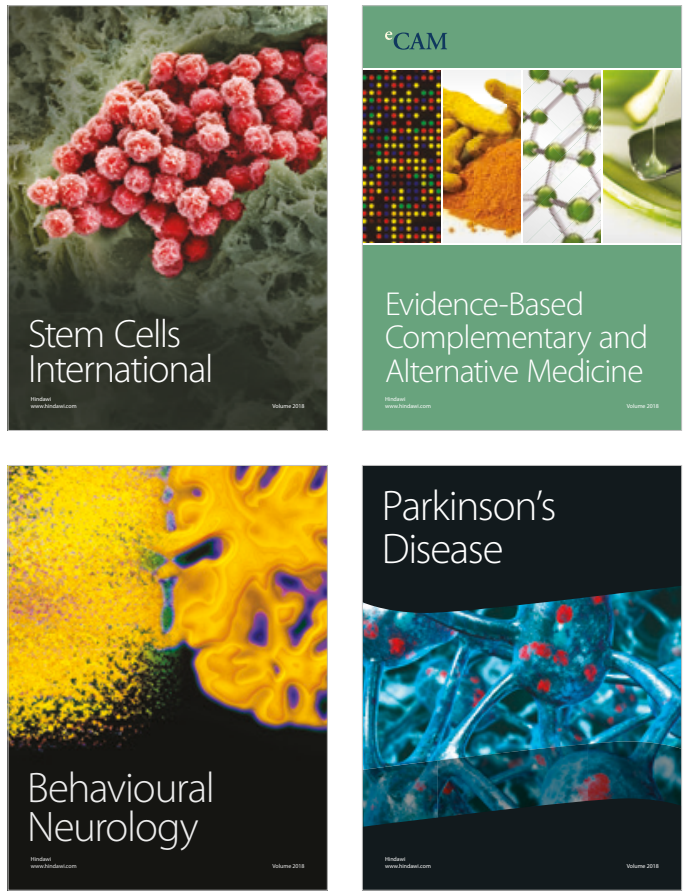

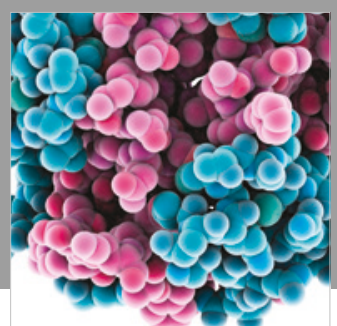

ournal of

Diabetes Research

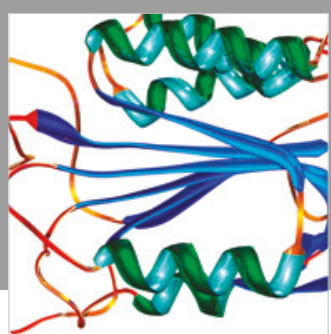

Disease Markers
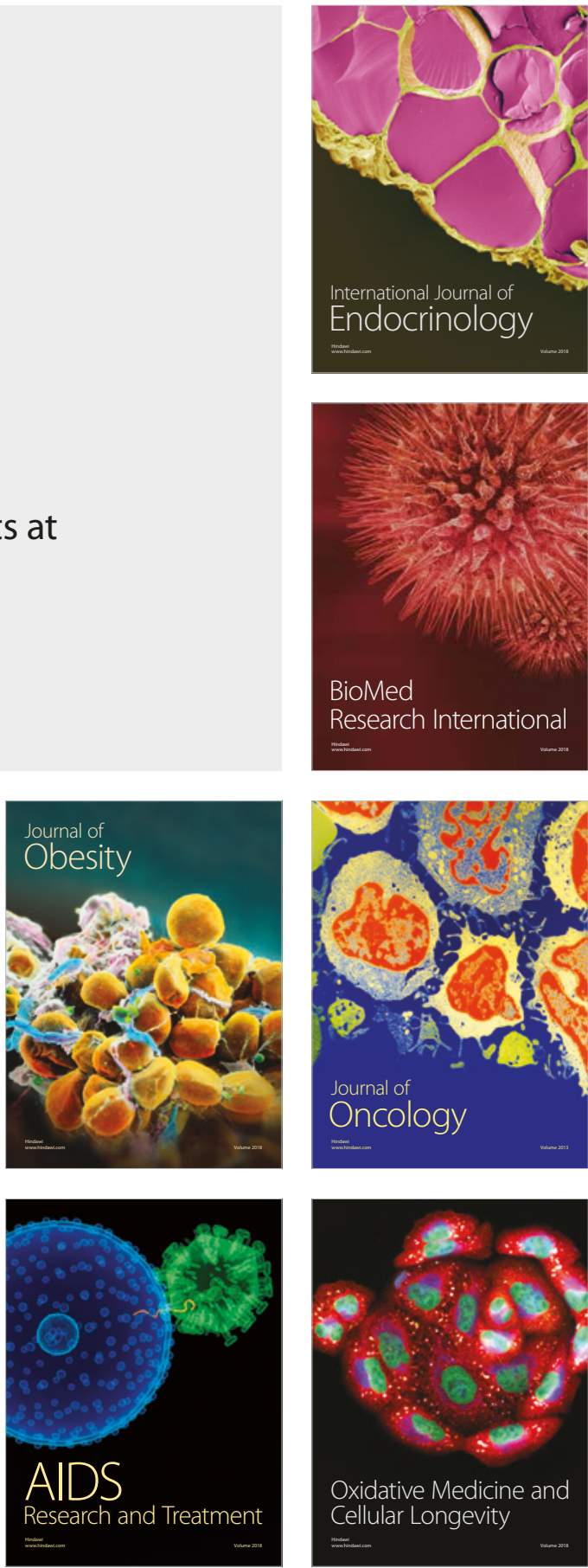